\title{
Emerging Trends in Hybrid Cloud and the Race among Global Leaders
}

\author{
Mirdul Swarup \\ High school student \\ Selaqui International School, India
}

\begin{abstract}
Over the years the IT world has changed a lot. With the introduction of new technology, the IT sector has presented a totally new face to the world. Cloud computing was introduced way back in 1990's. But it came in light in $21^{\text {st }}$ century. Many surveys by many international organizations have been done. And which are reviewed in this paper. This review paper also highlights a comparative study between many companies. It also highlights various cloud services, deployment models and also the main characteristics. There is a difference which is also stated in this between various other paradigms which seems to be like cloud environment.
\end{abstract}

\section{Keywords}

Cloud computing, cloud services, deployment models, comparative study

\section{INTRODUCTION}

Computing designed like normal commodities like traditional utilities. In such a way that users can access like the way they want and from anywhere they want. Cloud computing is an IT paradigm (model) which relies on sharing of resources to reduce investment in individual setups so that companies can gain profit at an economic scale from their IT department .In cloud environment the end user pay for the services they choose, cutting of the extra expenses on hardware and infrastructures and the rest of the managing and security issues are managed by the service provider .It is a conceptual based technology which is widely used nowadays [1][2].

After the launch of EC-2 in 2006 cloud computing environment has evolved and transformed the way how companies work because now they don't have to invest much in their IT sectors. All they have to do is to deploy a server in the cloud environment and use the "pay as you go" service according to their convenience [4].

Basically, the data and definitions provided by the NIST, the cloud consist of 5 characteristics, 4 deployment models and 3 types of services.

But except all the flexibility and scalability cloud computing arena faces a lot of privacy issues and challenges. There are trust problems between service providers and consumer and other issues like privacy leaks, cloud intrudes etc. But to reduce security issues many organizations have been set up such as Akamai etc. To maintain the "Quality of service" (qos).[3]

\section{CHARACTERISTICS OF CLOUD}

After examining its difference from other paradigms, the cloud computing offers some of its characteristics. In which NIST laid on five of its essential characteristics [5]-

2.1 On demand self-service - Consumer can provision such as processing power, server time, and network without any human intervention.
2.2 Broad network services - Clients can access the service by any thick or thin client platforms (e.g.-workstations, web browsers, phones, laptops etc.)

2.3 Resource pooling - These characteristics explains the concept of multi tenancy as customers share pooled computer resources. Because the service provider's computing resources are used to serve multiple clients. Cloud consumer can specify where to store data at geographical level. But the client will have no idea about the location of the application or data storage.

2.4 Rapid elasticity - Prominent capabilities like storage network bandwidth and computer resources availed by the client can be elastically provisioned and released automatically to scale outward and inward and match the demands. The services can be applied in any quantity at any time.

2.5 Measured service- Cloud has the leveraging metering capability that can actually measure transactions, bandwidth, active users and the utilization of resources and in addition they monitor the control of resources. And all this provides transparency for both service provider and the client.

\section{CLOUD COMPUTING A TOTALLY DIFFERENT ARENA}

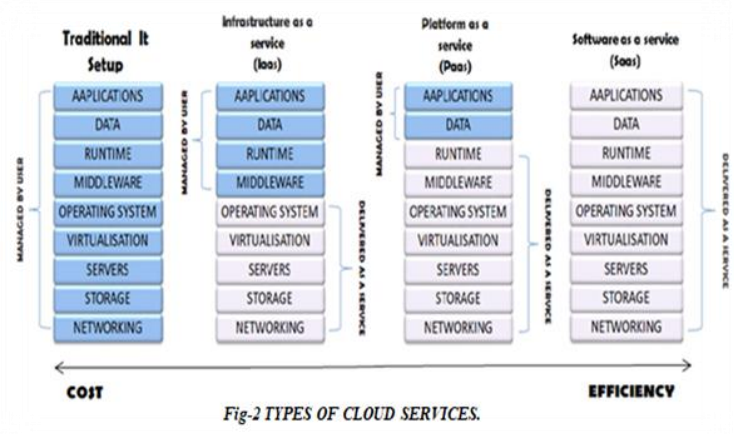

Fig-1-Growth of cloud computing 2015-2018

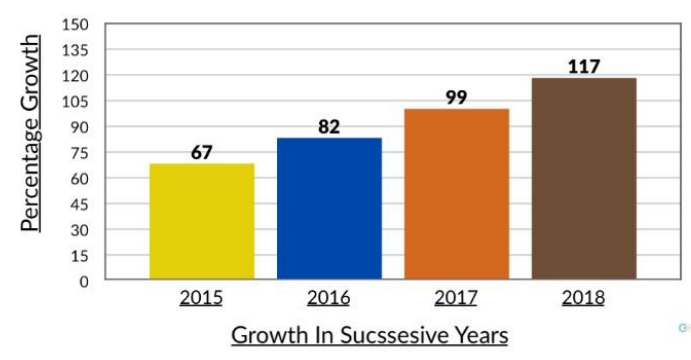


Mostly cloud computing is confused to be a combination of cluster and grid computing. However, cloud computing is just a next generation data center. Including the features of both cluster and grid computing. Clouds have the hardware of different grids whereas software to that of cluster.

The clouds environment proved to be more flexible and scalable than other paradigms in same arena. As it is

Fig-3 $-95 \%$ of respondants are using cloud!

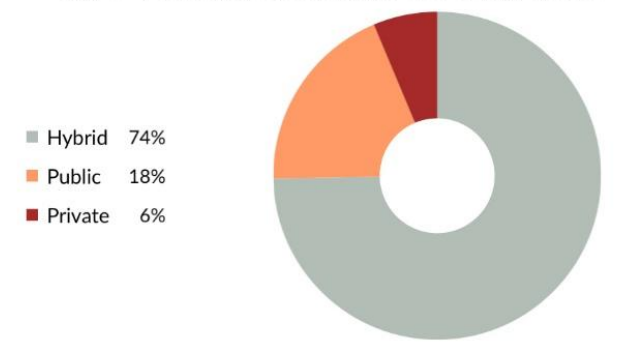

considered to be the advance level of all its companion paradigms, it is more feasible from cluster and grid computing because of its main feature of multitenancy which makes it quite a totally different sector in which companies can earn as well as save their thousands of grands [6].

Cloud computing is also ahead of other paradigms as it covers a major larger area network (as shown in fig-1) .

As for consideration cluster can be compared with LAN, grid computing with MAN and clouds as WAN. And as it is so wide that the client can use it from anywhere and on any device, they want [8][9].

Through years the popularity of different paradigms has also varied as the trend measures the popularity of all the three computing environments as during 1990's cluster computing was buzz word, from early 2000 grid computing came in light and overcoming it cloud computing changed networking and IT management for companies. After the introduction of the concept of cloud computing many multinational companies launched their cloud services making currently AWS (Amazon Web Services), Azure, Cloud Platform and Alibaba global leaders in it.

\section{TYPES OF CLOUD SERVICES}

The cloud computing also provides three types of service models from which the users can choose which ever service they want according to their requirements which are stated as follows (as shown in fig-2) -

\section{Infrastructure As A Service (IAAS)}

In this service model, the server provides client with computing infrastructure which includes resources such as network, operating server, and storage using the virtual technology concept. This service is highly scalable and uses automated computer resources. After getting provided with everything it may depend on the service provider, that they might be able to configure their networking but in more sophisticated and limited way. The biggest advantage is that the companies have the same capabilities and technologies as their traditional data centers only the difference is that now their data centers are now "virtual data centers" in the cloud environment [10].I

\section{Where a client can use IAAS}

There are certain set of conditions in which each service model is used as IAAS is mostly preferred for the small companies and startups, as the startups can't invest much in their infrastructure and have to earn profits for their initial periods. it is also beneficial for the large companies which don't want to spend their time, money and labour in trying to create their software and hardware [11].

\section{Platform As A Service (PAAS)}

Enterprises use PAAS to deploy and maintain applications, while other things are observed and maintained by the service provider such as the middleware and the operating server. this delivery service gives liberty to the user for making software without having an eye on the other essentials such as software issues, updates, infrastructure and the storage problems. But somewhat PAAS lacks behind from IAAS in this [3].

\section{Where a client can use PAAS}

Mostly it is more flexible and successful if there are multiple workers and developers on single projects. PAAS is generally more scalable if some other client has to be added on the same project. It is majorly profitable in cases where the apps have to be rapidly made or deployed.

\section{Software As A Service (SAAS)}

It is one of the most familiar cloud delivery models and also can be called as cloud application services. This model usually uses the net services to provide its applications to its clients and these are mostly managed by the third-party vendors. In this model service the clients do not need any installation on any client side as the software and applications can be accessed by the web services. And also, for the ease (Placeholder1)the companies are rarely responsible for much except some of the data and configuration management. It can also be referred as "on demand software" because in this the user have to only pay for the usage according to his/her requirements [12].

\section{Where a client we can use SAAS}

It can be majorly for the software and applications which aren't used more often, or which are not much in demand. It is majorly for the enterprises which are usually in collaboration or in joint ventures for short duration.

\section{DIFFERENT TYPES OF DEPLOYMENT MODELS}

\section{Public cloud}

When the cloud infrastructure and services are made available to a large industry or group. It provides global scale and intensive capabilities for example- networks, and savings through economies. So that the operations can be accomplished with more flexibility and efficiency .It is usually based on demand pay per as use or can be free .Major advantage of public cloud is that it helps to reduce cost over many things and they are usually managed by the datacenters which belongs to the service providers and this model use the concept of multi-tenancy which means the resources provided by the service providers is used by the multiple customers [13].

\section{Advantages of public cloud}

5.1 Clients can create their own clouds if they do not want to depend on the party.

5.2 We can access it from anywhere and from any device we want according to our ease.

5.3 The client only must pay for the services the client has applied for. 


\section{Private cloud}

Private cloud mainly serves one particular organization but however unlike the public cloud it lacks the capability and

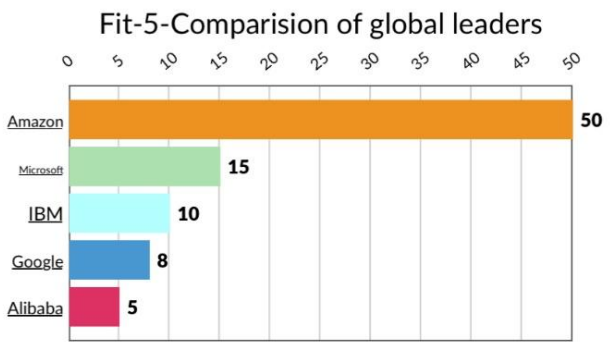

Worldwide Share - 2017

economies of scale. As it is shared by one organization so there is no interference of the outsiders. so, this feature makes it more secure but on the more it makes less flexible which is one of the main features of the public clouds. But the organizations concerned about their security can be burden free as the private cloud paradigm are not to prone malicious. attacks. It also uses a "pay per use model" to cater spikes in demand of cloud computing [13].

\section{Advantages of private cloud}

5.1 As it serves only a particular organization so the organizations can modify the clouds according to their requirements.

5.2 As the private clouds are made only for a particular organization so the security is at its level best which the other cloud deployment models lack.

5.3 As the organizations have their own cloud systems so there is no compromise in the resources.

\section{Hybrid cloud}

The recent success of this cloud deployment model has made it the most flexible and versatile paradigm from which the enterprises are earning huge profits without investing their much revenue in IT infrastructure. As it has the features of both private and public deployment models. Hybrid cloud is a mix of environments of both the other models is called cloud bursting and it enables highly elastic environments. And this paradigm also offers access to data and application. Hybrid clouds are nowadays the most popular service model [13].

\section{Advantages of hybrid cloud}

5.1 It is more compatible as client have to only pay for the extra expenses

5.2 Hybrid cloud is much more versatile and flexible than other two models.

5.3 It highly profitable to organization of every size and of any sector as it has the feature of many other model

\section{EMERGENCE OF HYBRID CLOUD!}

Throughout the paper there was a discussion about the cloud, its characteristics, its services, its characteristics, services and models. But from more than half a decade there is a particular sector within cloud computing which is at its peak which has affected both the service provider and the clients. And this sector is known as "hybrid cloud". (as shown in fig-3) [13].

As the name suggests it is a mix of other deployment models and can be said as "the best of both the private and public worlds" because the main advantage of hybrid cloud is, it provides environment which is a mix of on premises private cloud and a third party with the combination of services of orchestration between the two platforms which is a feature of

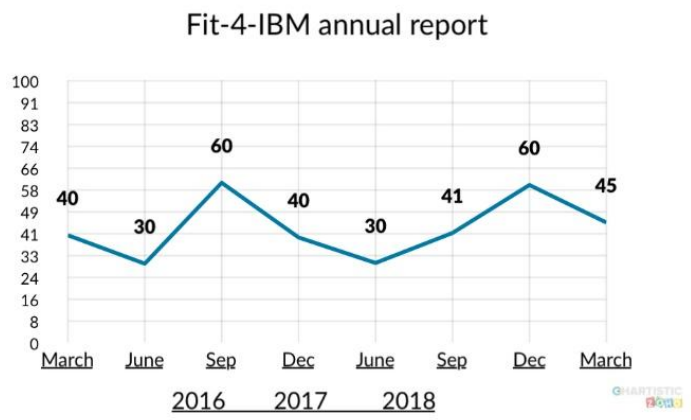

public clouds. Many companies tried to draw out an algorithm to help the customer for choosing an appropriate cloud deployment model. But the outcomes were only in the favor of hybrid clouds because of its mixed environment making it the most flexible, scalable and cost-effective model. Over the years companies noticed variations in many cloud services. But they saw a common trend of growing services in hybrid clouds [14].

Many companies have their individual reports of their sales growth in hybrid clouds. But moving further there is a comparative study of case studies of data, client list, revenue reports provided by the companies which are global leaders in their respective services whether it be the service provider or the clients such as AWS, Gartner, Azure, IBM, Redpixie, Alibaba and many others [14].

The case studies and reports released by IBM gives a rough idea of hybrid clouds have affected their business. Furthermore, are the transformation study that states [15] [16] (as shown in fig-4)-

6.1 The figures show that 8 out of 10 leaders believe that hybrid clouds are most secure.

6.2 Global leaders are using hybrid models because its real time analytics to manage hybrid environments.

$6.362 \%$ percent of businessmen believe hybrid helps them to meet compliance requirements.

Similarly, one of the multinational company REDPIXIE published a booklet according to the information and definitions provided by Gartner there is a clear mention that in upcoming years this global shift towards the cloud will affect $£ 796$ billion in IT expenditure and looking at the trends and statistics this is possible only because of the investments done by companies in hybrid clouds [14].

Observing the other major players such as AWS (Amazon Web Services) which has earned the most through cloud computing. They had also provided their case studies which leaves no doubt on the fact that small companies are emerging as multi nationals by saving their millions of dollars in IT structures. Because of their investments in clouds and majorly the hybrid ones.

AWS client list also includes major players which have also deployed hybrid cloud and today are at the top of their sectors [17].

Many companies have also stated that its favorable to invest in hybrid ones as it provides you the exposure to that of a 
public cloud in addition security and privacy of a private cloud.

\section{COMPETITION BETWEEN GLOBAL LEADERS FOR FIRST PLACE}

Cloud environment has made millions of dollars for many companies, looking at the graphs currently Amazon Web Services has taken the first place having billions of dollars as their net worth (as shown in fig-5).

But other enterprises such as Azure, Google Cloud Platform and Jack Ma's Chinese based Alibaba cloud are pushing it hard to pull down AWS from its place. After observing dozens of case studies. Amazon serves major dominating companies in market such as Uniliver, Instagram etc. But after a $1 \$$ billion investments in $2015 \mathrm{Ma}$ 's Alibaba has annual growth percentage of $126.5 \%$ in their 2016-2015 final quarters. And by this investment they have opened their centers in United States and in Europe in 2016[22].

\section{COMPARISION BETWEEN EC2 AND ECS}

Reports have clearly shown that EC2 and ECS have all different instances. So, a pricing comparison cannot be done between these two. The main difference between these two cloud environments is Alibaba is still confined to Asian regions and AWS has a larger geographic coverage. There is also a slight difference between both of these companies pricing models, as Alibaba cloud uses a "pay-as-you-go" model whereas Amazon gives "on demand pricing". Cloud storage services of Alibaba has three tiers for its operating system service [18]-

\subsection{Standard \\ 8.2 Infrequent access}

8.3 Achieve

Amazon also have a similar offering, but comparatively Alibaba wins the race as their storage costs are slightly lower than those of AWS. Analysts have also stated that Alibaba must reach the trillion-dollar mark by 2020 .

Both of these can also be compared on the basis of community and awareness. AWS has a majority in western countries and especially people who speak English whereas Alibaba is still not that prominent in Western areas.

Amazon dominates North America, but Alibaba have its roots in Chinese markets. But China's current economic growth. But china's current economic growth provides faster and longer runway to Alibaba [22].

\section{COMPARISION BETWEEN CLOUD PLATFORM AND AWS}

Google's Cloud Platform and Amazon's AWS can be compared on various measures such as the service they provide. Comparisions can be done on the features they provide. Both the companies provide very similar ways to handle IP address. In the economic growth, Cloud Platform had a growth percentage of $100 \%$ in the year 2015-2016 as compared to Amazon with a growth percentage as $45.9 \%$. If we investigate instance migration companies removed their host machines for maintenance or replacement. Cloud Platform provides live migration in which Cloud Platform automatically and transparently migrates their instances when there is a need of any modifications. In contrast, in AWS the user has to manually migrate instance from these host machines, either by rebooting or recreating. Google Cloud Platform has made a tough competition their quarterly revenues are also increasing in successive years. But Bezos's compassion of making Amazon the most "customer centric companies" is still on the top. Observing the peering services. Amazon provides direct connect which allows clients to create a private leased to AWS. Google also gives carrier interconnect which allows the clients to create a private leased line into Cloud Platform from a partner facility. Google's carrier interconnect connects the client's traffic to global network rather to specific region [19] [20] [23].

\section{CONCLUSION}

In this review paper there are stats and reports about cloud computing and how it is changing the IT world. At first characteristics are stated which mainly explains the features of cloud computing and it is different from other computing models which are considered to be similar as cloud. Definitions about the various deployment models and the various types of services that cloud environment provides are also stated. later on, there is a justification of how hybrid cloud is one of the most versatile deployment model. Reports and stats have shown that in the upcoming years hybrid cloud will totally transform the way multinational companies do their business. Moving on further there is a review about the on-going competition between major leading companies such as Amazon, Alibaba and Google for the first place in the cloud business and the observing the surveys by many organisations a result can be concluded that Alibaba and Google might pull down Amazon from its place as both the other companies have growth percentage way more than Amazon. But Amazon is also trying it hard to maintain their place as they have also entered the Chinese market and more Asian regions. This review paper shows a clear vision of cloud computing and the global leaders of transforming the world with this new buzzword called "cloud computing".

\section{ACKNOWLEDGEMENT}

My thanks to the experts who have contributed in my research and supported me. A special thanks to my computer professor Dr.Ashish Vashistha, my mentor Mr. Itihaas Singh and our Headmaster Mr. Rashid Sharfuddin .

\section{REFERENCES}

[1] Janakiram MSV cloud computing strategist ;(2010), "demystifying the cloud an introduction to cloud computing", version 1.0- march.

[2] Geng L; David F ; Jinzy J; Glenn D ; (2009) "cloud computing IT as a service ", IEEE computer society IT professional”. Vol.11, pp 10-13 March-April 2009.

[3] Pankaj Arora, Raj Biyani, Salil Dave, "Explore" in "To the cloud", New Delhi, India : Tata Mc Graw Hill Edition,2011.

[4] itinfo.am[online].Available: http://www.itinfo.am/eng/cloud-computing

[5] Petre Mell,Tim Grance,'DRAFT NIST WORKING

[6] DEFINITIONS,"[ONLINE]Available:http://csrc.nist.gov /grps/SNS/cloud computing .

[7] Tian LQ; NI Y, LING;(2010)," Evolution of user behavior trust in cloud computing "2010 international conference on computer applications and system modelling (ICCASM 2010), Vol. 7, pp V7-567,22-24 oct 2010 . 
[8] Mathur, P; Nishchal , N.;(2010), "cloud computing new challenges to the entire computer industry ", $20101_{\text {st }}$ international conference on parallel, distributed and grid computing (PDGC-2010),pp 223.

[9] techopedia.com[online].Available: https://www.techopedia.com/definition/87/gridcomputing

[10] techopedia.com[online].Available: https://www.techopedia.com/definition/6581/computercluster

[11] "Infrastructure as a Service Security: Challenges and Solutions ", Wesam Dawoud Ibrahim Takouna, Christoph Meinel, Hasso, Plattner Institute.

[12] STATECHMAGZINE.COM. [online]. Available: http://www.statechmagzine.com/article/2014/03/5important -benefits-infrastructure- service.

[13] "Implementation of SaaS in cloud computing environment" Venkata Rao J. and D. Bhargava Reddy, Department of CSE, K.L. University, Vaddeswaram, Guntur Andhra Pradesh, India, International Journal of Computer Sciences and Telecommunications, Vol. 2, Issue 8, November 2011.

[14] Parsi, K., \& M.Laharika .(2013) "A comparative study of different deployment models in a cloud" International Journal of Advanced Research in Computer Science and Software Engineering , 3 (5), 512-515.

[15] REDPIXIE.COM.[online].Available: http://info.redpixie.com/hubfs/Hybrid-cloud-guide/.

[16] IBM.COM.[online].Available: https://www.ibm.com/cloud-computing/bluemix/casestudies.

[17] IBM.COM.[online].Available: https://www.ibm.com/blogs/cloud-computing/tag/clientstories/.

[18] AMAZON.COM.[online].Available: https://aws.amazon.com/solutions/case-studies/all/.

[19] AMAZON.COM.[online].Available: https://aws.amazon.com.

[20] CLOUD ACADEMY.COM. [online].Available: Https://cloudacademy.com/blog/ec2-vs-google-computeengine/.

[21] CLOUD ACADEMY.COM. [online].Available: Https://cloudacademy.com/blog/google-cloud-vs-aws-acomparison/.

[22] CONTINO.COM[online].Available: https://www.contino.io/insights/whos-using-aws.

[23] therobinreport.[online].Available: https://www.therobinreport.com/alibaba-vs-amazonwho-will-become-biggest-fastest/.

[24] GOOGLE.COM.[online].Available: https://cloud.google.com.Mirdul Swarup is a high school student in Selaqui International School. He is currently preparing for the All India Senior School Certificate Examination. $\mathrm{He}$ is an avid programmer. Mirdul is planning to attend a top university and pursue his career in computer science and engineering. His research interest lies in cloud computing and database. 